\title{
Gradient estimate of a Neumann eigenfunction on a compact manifold with boundary
}

\author{
Jingchen Hu, Yiqian Shi and Bin Xu
}

\begin{abstract}
Let $e_{\lambda}(x)$ be a Neumann eigenfunction with respect to the positive Laplacian $\Delta$ on a compact Riemannian manifold $M$ with boundary such that $\Delta e_{\lambda}=\lambda^{2} e_{\lambda}$ in the interior of $M$ and the normal derivative of $e_{\lambda}$ vanishes on the boundary of $M$. Let $\chi_{\lambda}$ be the unit band spectral projection operator associated with the Neumann Laplacian and $f$ a square integrable function on $M$. We show the following gradient estimate for $\chi_{\lambda} f$ as $\lambda \geq 1:\left\|\nabla \chi_{\lambda} f\right\|_{\infty} \leq \mathrm{C}\left(\lambda\left\|\chi_{\lambda} f\right\|_{\infty}+\lambda^{-1}\left\|\Delta \chi_{\lambda} f\right\|_{\infty}\right)$, where $C$ is a positive constant depending only on $M$. As a corollary, we obtain the gradient estimate of $e_{\lambda}$ : for every $\lambda \geq 1$, there holds $\left\|\nabla e_{\lambda}\right\|_{\infty} \leq \mathrm{C} \lambda\left\|e_{\lambda}\right\|_{\infty}$.
\end{abstract}

Mathematics Subject Classification (2000): Primary 35P20; Secondary 35J05

Key Words: Neumann eigenfunction, gradient estimate

\section{Introduction}

Let $(M, g)$ be an $n$-dimensional compact smooth Riemannian manifold with smooth boundary $\partial M$ and $\Delta$ the positive Laplacian on $M$. In local coordinate chart $x=\left(x_{1}, \cdots, x_{n}\right)$, $\Delta$ can be expressed by

$$
\Delta=-\frac{1}{\sqrt{g}} \sum_{i, j} \partial_{x_{i}}\left(g^{i j} \sqrt{g} \partial_{x_{j}}\right),
$$

where $\left(g^{i j}\right)=\left(g^{i j}(x)\right)$ is the inverse of the metric matrix $\left(g_{i j}\right)=\left(g_{i j}(x)\right)=g\left(\partial_{x_{i}}, \partial_{x_{j}}\right)$, and $\sqrt{g}=\sqrt{g(x)}:=\operatorname{det}\left(g_{i j}(x)\right)$. In this paper, we always mean doing the summation from 1 to $n$ when we omit the variation domain of indices. Let $L^{2}(M)$ be the space of square integrable functions on $M$ with respect to the Riemannian density $d V=\sqrt{g(x)} d x$. Let $e_{1}(x), e_{2}(x), \cdots$ be a complete orthonormal basis in $L^{2}(M)$ for Neumann eigenfunctions of $\Delta$ such that $0=\lambda_{1}^{2}<\lambda_{2}^{2} \leq \lambda_{3}^{2} \leq \cdots$ for the corresponding eigenvalues, where $e_{j}(x)$ $(j=1,2, \ldots)$ are real valued smooth function on $M$ and $\lambda_{j}$ are nonnegative numbers.

The second author is supported in part by the National Natural Science Foundation of China (No. 10671096, No. 10971104), the third author by the National Natural Science Foundation of China (No. 10601053, No. 10871184). All of the authors are supported in part by the Fundamental Research Funds for the Central Universities. 
Also, let $\mathbf{e}_{j}$ denote the projection of $\mathrm{L}^{2}(\mathrm{M})$ onto the 1-dimensional space $\mathbf{C} e_{j}$. Thus , an $L^{2}$ function $f$ can be written as $f=\sum_{j=0}^{\infty} \mathbf{e}_{j}(f)$, where the partial sum converges in the $L^{2}$ norm. Let $\lambda$ be a positive real number $\geq 1$. We define the unit band spectral projection operator (UBSPO) $\chi_{\lambda}$ as follows:

$$
\chi_{\lambda} f:=\sum_{\lambda_{j} \in(\lambda, \lambda+1]} \mathbf{e}_{j}(f) .
$$

We call that this $\chi_{\lambda}$ is associated with the Neumann Laplacian on $M$. The corresponding UBSPO $\chi_{\lambda}$, where we use the same notion, can also be defined for both the Dirichlet Laplacian on $M$ and the Laplacian on a closed Riemannian manifold.

Grieser [7] and Sogge [15] proved the following $L^{\infty}$ estimate on $\chi_{\lambda}$ associated with the Dirichlet Laplacian,

$$
\left\|\chi_{\lambda} f\right\|_{\infty} \leq C \lambda^{(n-1) / 2}\|f\|_{2}
$$

where $\|f\|_{r}(1 \leq r \leq \infty)$ means the $L^{r}$ norm of the function $f$ on $M$. In the whole of this paper $C$ denotes a positive constant which depends only on $M$ and may take different values at different places unless otherwise stated. The idea of Grieser and Sogge is to use the standard wave kernel method outside a boundary layer of width $C \lambda^{-1}$ and a maximum principle argument inside that layer. Using their idea, $\mathrm{Xu}$ [18] proved estimate (1) for the Neumann Laplacian. On the other hand, Hart F. Smith [14] proved a sharp $L^{2} \rightarrow L^{p}$ estimate for $\chi_{\lambda}$ on a closed manifold with Lipschitz metric. As a consequence, (1) holds for both the Dirichlet or Neumann Laplacian provided $\operatorname{dim} M=2$ or 3 .

By using the maximum principle argument and the estimate (1), $\mathrm{Xu}[17,18]$ proved the following gradient estimate on $\chi_{\lambda}$ for both the Dirichlet and Neumann Laplacian,

$$
\left\|\nabla \chi_{\lambda} f\right\|_{\infty} \leq \mathrm{C} \lambda^{(n+1) / 2}\|f\|_{2}
$$

Here $\nabla$ is the Levi-Civita connection on $M$. In particular, $\nabla f=\sum_{j} g^{i j} \partial f / \partial x_{j}$ is the gradient vector field of a $C^{1}$ function $f$ in a local coordinate chart $\left(x_{1}, \cdots, x_{n}\right)$, the square of whose length equals $\sum_{i, j} g^{i j}\left(\partial f / \partial x_{i}\right)\left(\partial f / \partial x_{j}\right)$. One of his motivation is to prove the Hörmander multiplier theorem on compact manifolds with boundary. Seeger and Sogge [11] firstly proved that theorem on closed manifolds by using the parametrix of the wave kernel. Duong-Ouhabaz-Sikora [5] proved a general spectral multiplier theorem on closed manifolds by the $\mathrm{L}^{2}$ norm estimate of the kernel of spectral multipliers and the Gaussian bounds for the corresponding heat kernel. As an application, they gave an alternative proof to the Hörmander multiplier theorem on closed manifolds by using the $\mathrm{L}^{\infty}$ estimate (1) of $\chi_{\lambda}$ and the heat kernel.

By rescaling $\chi_{\lambda} f$ at the scale of $\lambda^{-1}$ both outside and inside the boundary layer of width $C \lambda^{-1}$, for $\chi_{\lambda}$ associated with the Dirichlet Laplacian, the last two authors [13] obtained 
by elliptic a priori $C^{1, \alpha}$ estimates the following estimate slightly finer than (2),

$$
\left\|\nabla \chi_{\lambda} f\right\|_{\infty} \leq \mathrm{C}\left(\lambda\left\|\chi_{\lambda} f\right\|_{\infty}+\lambda^{-1}\left\|\Delta \chi_{\lambda} f\right\|_{\infty}\right), \quad f \in L^{2}(M) .
$$

See $[13$, Remark 1.2] for the argument that the above estimate with the help of (1) could imply the Dirichlet case of estimate (2) by Xu. On the other hand, an immediate consequence of our estimate (3) is as follows: there exists a constant $C$ such that for each Dirichlet eigenfunction $e_{\lambda}$, i.e., $\Delta e_{\lambda}=\lambda^{2} e_{\lambda}$ in the interior of $M$ and $e_{\lambda}=0$ on the boundary of $M$, we have $\left\|\nabla e_{\lambda}\right\|_{\infty} \leq C \lambda\left\|e_{\lambda}\right\|_{\infty}$. Furthermore, following the idea of Brüning [2] and Zelditch [19, Theorem 4.1], the last two authors ([13, Lemma 2.2]) proved a basic geometry property of nodal sets for Dirichlet eigenfunctions, i.e. as $\lambda$ sufficiently large, every geodesic ball with radius $C / \lambda$ and lying in the interior $\operatorname{Int}(M)$ of $M$ must contain at least one zero point of a Dirichlet eigenfunction with eigenvalue $\lambda^{2}$. We call this the equidistribution property of a non-trivial Dirichlet eigenfunction, using which we obtained a two-sided gradient estimate for a non-trivial Dirichlet eigenfunction $e_{\lambda}$,

$$
C^{-1} \lambda\left\|e_{\lambda}\right\|_{\infty} \leq\left\|\nabla e_{\lambda}\right\|_{\infty} \leq \mathrm{C} \lambda\left\|e_{\lambda}\right\|_{\infty} \text { for all } \lambda \geq 1
$$

In the paper, we obtain in part the Neumann version of results by the last two authors.

Theorem 1.1. Let $f$ be a square integrable function on the compact Riemannian manifold $(\mathrm{M}, \mathrm{g})$ with boundary $\partial \mathrm{M}$. Let $\chi_{\lambda}$ be the UBSPO associated with the Neumann Laplacian. Then, for all $\lambda \geq 1$ and for all $f \in \mathrm{L}^{2}(M)$, there holds

$$
\left\|\nabla \chi_{\lambda} f\right\|_{\infty} \leq \mathrm{C}\left(\lambda\left\|\chi_{\lambda} f\right\|_{\infty}+\lambda^{-1}\left\|\Delta \chi_{\lambda} f\right\|_{\infty}\right) .
$$

In particular, letting $f=e_{\lambda}(x)$ be an eigenfunction with respect to the positive Neumann Laplacian on $M$, i.e., $\Delta e_{\lambda}=\lambda^{2} e_{\lambda}$ in the interior of $M$ and the normal derivative of $e_{\lambda}$ vanishes on the boundary of $M$, we obtain

$$
\left\|\nabla e_{\lambda}\right\|_{\infty} \leq \mathrm{C} \lambda\left\|e_{\lambda}\right\|_{\infty}
$$

Remark 1.1. We shall prove Theorem 1.1 directly via maximum principle argument in Section 3. It is quite different from that of the Dirichlet case (3) in [13, Section 3], where the last two authors used the $\mathrm{C}^{1, \alpha}$ a priori estimate. Moreover, our maximum principle argument in this paper would NOT go through for the Dirichlet case. Heuristically speaking, we should owe the success of the maximum principle in the Neumann case to 
Fact 1 If a $\mathrm{C}^{2}$ function $\mathrm{g}$ on the half real line $[0, \infty)$ satisfies $\mathrm{g}^{\prime}(0)=0$, then the even extension of $\mathrm{g}$ is also $\mathrm{C}^{2}$ on the real line $(-\infty, \infty)$.

Our failure of using the maximum principle argument in the Dirichlet case is partially due to

Fact 2 If a $C^{2}$ function $h$ on the half real line $[0, \infty)$ satisfies $h(0)=0$, then the odd extension of $\mathrm{h}$ to the real line $(-\infty, \infty)$ is NOT $\mathrm{C}^{2}$ on $(-\infty, \infty)$ in general.

Precisely speaking, by Fact 1, we can reduce the gradient estimate (5) near the boundary to the interior case, which will be proved by the standard maximum argument combined with the frequency dependent rescaling technique. However, Fact 2 prevents us from doing the similar thing for the Dirichlet case.

Remark 1.2. Theorem 1.1 strengthens the Neumann case of estimate (2) proved by $\mathrm{Xu}[18]$ in the sense that it shows how the gradient estimate on a Neumann eigenfunction depends on its supremum. In particular, the similar argument as [13, Remark 1.2] shows that estimate (5) together with (1) imply the Neumann case of estimate (2) by Xu [18]. However, (2) is strong enough for $\mathrm{Xu}$ to prove his Hörmander multiplier theorem associated with the Neumann Laplacian on $M$. The authors' motivation is to prove the Neumann version of the result of Shi-Xu [13].

Remark 1.3. We conjecture that each Neumann eigenfunction has the equidistribution property, i.e. every geodesic ball with radius $\mathrm{C} / \lambda$ and lying in the interior $\operatorname{Int}(M)$ of $M$ must contain at least one zero point of a Neumann eigenfunction with eigenvalue $\lambda^{2}$. If it were true, then we could prove the following lower bound estimate

$$
\left\|\nabla e_{\lambda}\right\|_{\infty} \geq C \lambda\left\|e_{\lambda}\right\|_{\infty}
$$

by a little modification of the argument in [13, Section 2]. However, the idea of the proof for the equidistribution property of a non-trivial Dirichlet eigenfunction in $[13$, Section 2$]$ did not go through for a Neumann eigenfunction, because the restriction of a Neumann eigenfunction to one of its nodal domains only satisfies the mixed Dirichlet-Neumann boundary condition in general.

We conclude the introduction by explaining the organization of the left part of this paper. We use the even extension and the maximum principle to show Theorem 1.1 (5), which implies the upper bound of $\nabla e_{\lambda}$. We also provide an alternative proof of Theorem 1.1 by the same even extension and the $C^{1, \alpha}$ a priori estimate. 


\section{Estimate for gradient of eigenfunction}

\subsection{Outside the boundary layer}

Recall the principle: On a small scale comparable to the wavelength $1 / \lambda$, the eigenfunction $e_{\lambda}$ behaves like a harmonic function. It was developed in $\mathrm{H}$. Donnelly and C. Fefferman $[3,4]$ and was used extensively there. In this section, for a square integrable function $f$ on $M$, letting $\chi_{\lambda}$ be the UBSPO associated with the Neumann Laplacian, we shall give a modification of this principle, which can be applied to the Poisson equation

$$
\Delta \chi_{\lambda} f=\sum_{\lambda_{j} \in(\lambda, \lambda+1]} \lambda_{j}^{2} \mathbf{e}_{j}(f) \text { in } \operatorname{Int}(M)
$$

with the Neumann boundary condition satisfied by $\chi_{\lambda} f$ on $\partial M$. Moreover, in this subsection, we only do analysis outside the boundary layer $L_{1 / \lambda}=\{z \in M: d(z, \partial M) \leq 1 / \lambda\}$ of width $1 / \lambda$.

Take a point $p$ with $d(p, \partial M) \geq 1 / \lambda$. We may assume that $1 / \lambda$ is sufficiently small such that there exists a geodesic normal coordinate chart $\left(x_{1}, \ldots, x_{n}\right)$ on the geodesic ball $B\left(p, \frac{1}{2 \lambda}\right)$ in $M$. In this chart, we may identify the ball $B\left(p, \frac{1}{2 \lambda}\right)$ with the $n$-dimensional Euclidean ball $\mathbb{B}\left(\frac{1}{2 \lambda}\right)$ centered at the origin 0 , and think of the function $\chi_{\lambda} f$ in $B\left(p, \frac{1}{2 \lambda}\right)$ as a function in $\mathbb{B}\left(\frac{1}{2 \lambda}\right)$. Our aim in this subsection is to show the inequality

$$
\left|\left(\nabla \chi_{\lambda} f\right)(p)\right| \leq C\left(\lambda\left\|\chi_{\lambda} f\right\|_{L^{\infty}\left(\mathbb{B}\left(\frac{1}{2 \lambda}\right)\right)}+\lambda^{-1}\left\|\Delta \chi_{\lambda} f\right\|_{L^{\infty}\left(\mathbb{B}\left(\frac{1}{2 \lambda}\right)\right)}\right) .
$$

For simplicity of notions, we rewrite $u=\chi_{\lambda} f$ and $v=\Delta \chi_{\lambda} f$ in what follows. The Poisson equation satisfied by $u$ in $\mathbb{B}\left(\frac{1}{2 \lambda}\right)$ can be written as

$$
-\frac{1}{\sqrt{g}} \sum_{i, j} \partial_{x_{i}}\left(g^{i j} \sqrt{g} \partial_{x_{j}} u\right)=v .
$$

Consider the following rescaled functions

$$
\mathfrak{u}_{\lambda}(y)=\mathfrak{u}(y / \lambda) \quad \text { and } \quad v_{\lambda}(y)=v(y / \lambda) \quad \text { in the ball } \mathbb{B}(1 / 2) .
$$

The above estimate which we are after is equivalent to its rescaled version

$$
\left|\left(\nabla \mathfrak{u}_{\lambda}\right)(0)\right| \leq C\left(\left\|\mathfrak{u}_{\lambda}\right\|_{L^{\infty}(\mathbb{B}(1 / 2))}+\lambda^{-2}\left\|v_{\lambda}\right\|_{L^{\infty}(\mathbb{B}(1 / 2))}\right) .
$$

On the other hand, the rescaled version of the Poisson equation has the expression,

$$
\sum_{i, j} \partial_{y_{i}}\left(g_{\lambda}^{i j} \sqrt{g_{\lambda}} \partial_{y_{j}} u_{\lambda}\right)=-\lambda^{-2} \sqrt{g_{\lambda}} v_{\lambda}
$$

where

$$
g_{i j, \lambda}(y)=g_{i j}(y / \lambda), \quad g_{\lambda}^{i j}(y)=g^{i j}(y / \lambda) \quad \text { and } \quad \sqrt{g_{\lambda}}(y)=(\sqrt{g})(y / \lambda) .
$$


The last two authors [13, Section 3.1] proved (7) by the interior $C^{1, \alpha}$ estimate (cf Gilbarg-Trudinger [6, Theorem 8.32, p. 210]) for a second order elliptic equation of divergence type, where the $C^{\alpha}$ norm of coefficients $g_{\lambda}^{i j} \sqrt{g_{\lambda}}$ in the equation are involved. In the following paragraph, we shall give a different and more elementary proof of (7), where we use the maximum principle, however, the $C^{0,1}$ norm of coefficients $g_{\lambda}^{i j} \sqrt{g_{\lambda}}$ are involved. Note that the $C^{0,1}$ norms of $g_{\lambda}^{i j} \sqrt{g_{\lambda}}$ are uniformly bounded for all $\lambda \geq 1$.

For simplicity of notions, we set

$$
u_{\lambda}=\phi, \quad h=-\lambda^{-2} \sqrt{g_{\lambda}} v_{\lambda}, \quad a_{i j}=g_{\lambda}^{i j} \sqrt{g_{\lambda}}, \quad b_{i}=\sum_{j=1}^{n} \frac{a_{i j}}{\partial y_{j}} .
$$

Then the rescaled Poission equation (8) can be written as

$$
L \phi:=\sum_{i, j} a_{i j} \frac{\partial^{2} \phi}{\partial y_{i} \partial y_{j}}+\sum_{i} b_{i} \frac{\partial \phi}{\partial y_{i}}=h, \quad y \in \mathbb{B}(1 / 2) .
$$

We learned this maximum principle argument for proving (7) from Brandt [1, p. 95-6]. Moreover, we find that the constant-coefficient-assumption there could be removed. The idea is to construction a new function $\phi_{1}$ from $\phi$ of $n+1$ variables and apply the maximum principle to $\phi_{1}$. The details are as follows. Define

$$
\phi_{1}\left(y_{1}, \cdots, y_{n} ; z_{1}\right):=\frac{1}{2}\left(\phi\left(y_{1}+z_{1}, y_{2}, \cdots, y_{n}\right)-\phi\left(y_{1}-z_{1}, y_{2}, \cdots, y_{n}\right)\right)
$$

in the $(n+1)$-dimensional domain

$$
\mathscr{R}=\left\{\left(\mathrm{y}_{1}, \cdots, \mathrm{y}_{\mathrm{n}} ; z_{1}\right):|y|<1 / 4,0<z_{1}<1 / 4\right\}
$$

Writing

$$
\mathrm{L}_{1}=\mathrm{L}-\mu \frac{\partial^{2}}{\partial \mathrm{y}_{1}^{2}}+\mu \frac{\partial^{2}}{\partial z_{1}^{2}} \quad(\mu>0),
$$

we observe that, for sufficiently small $\mu$, this new operator is elliptic in the $n+1$ variables, and satisfies

$$
\left|\mathrm{L}_{1} \phi_{1}\right|=\left|\mathrm{L} \phi_{1}\right| \leq\|\mathrm{h}\| \quad \text { in } \mathscr{R},
$$

where we denote by $\|\cdot\|$ the $L^{\infty}$ norm in $\mathbb{B}(1 / 2)$. Choose a constant $C$ sufficiently large and depending on the $L^{\infty}$ norm of coefficients $a_{i j}$ and $b_{i}$ so that

$$
\mathrm{L}\left(|y|^{2}\right) \leq 2 \mu \mathrm{C}
$$

and introduce the comparison function

$$
\overline{\phi_{1}}:=\frac{1}{2 \mu}\|\mathrm{h}\|\left(\frac{1}{4} z_{1}-z_{1}^{2}\right)+16\|\phi\|\left\{|y|^{2}+z_{1}^{2}+C\left(\frac{1}{4} z_{1}-z_{1}^{2}\right)\right\} .
$$


Then we have

$$
\begin{aligned}
\mathrm{L}_{1} \overline{\phi_{1}} & =-\|\mathrm{h}\|+16\|\phi\|\left(\mathrm{L}\left(|y|^{2}\right)-2 \mu \mathrm{C}\right) \\
& \leq-\|\mathrm{h}\| \leq-\left|\mathrm{L}_{1} \phi_{1}\right| \text { in } \mathscr{R}
\end{aligned}
$$

and

$$
\overline{\phi_{1}} \geq\left|\phi_{1}\right| \quad \text { on the boundary } \partial \mathscr{R} \text {. }
$$

Thus, by the weak maximum principle (cf Gilbarg-Trudinger [6, Theorem 3.1, p. 32]), we obtain $\left|\phi_{1}\right| \leq \overline{\phi_{1}}$. This implies that

$$
\begin{aligned}
\frac{1}{2}\left|\phi\left(z_{1}, 0, \cdots, 0\right)-\phi\left(-z_{1}, 0, \cdots, 0\right)\right| & \leq \overline{\phi_{1}}\left(0, \cdots, 0, z_{1}\right) \\
& \leq \frac{1}{2 \mu} \frac{z_{1}}{4}\|\mathrm{~h}\|+16\|\phi\|\left(\frac{\mathrm{C} z_{1}}{4}+z_{1}^{2}\right) .
\end{aligned}
$$

Dividing through by $z_{1}$ and letting $z_{1} \rightarrow 0$ yields the desired estimate

$$
\left|\frac{\partial \phi}{\partial y_{1}}(0)\right| \leq \frac{1}{8 \mu}\|h\|+4 C\|\phi\| \text {. }
$$

Therefore, we complete the proof of (6).

We remark that (6) can also be proved directly by the above maximum principle argument without doing the re-scaling. Here we prefer to do the rescaling before proceeding to the maximum principle argument because of the following two reasons:

- Re-scaling makes the dependence relation of the desired estimate on the eigenvalue $\lambda^{2}$ clear and reduce the question to the case of a fixed scale.

- It is convenient for reader to compare the maximum principle argument here with the proof via the elliptic a priori estimate in Shi-Xu $[12,13]$.

\subsection{Inside the boundary layer}

Using the notions in subsection 2.1 , we are going to prove the following estimate:

$$
\left|\nabla u\left(p_{0}\right)\right| \leq C\left(\lambda\|u\|_{\infty}+\lambda^{-1}\|v\|_{\infty}\right) \quad \text { for all } p_{0} \in L_{1 / \lambda}
$$

with which combining (6) completes the proof of Theorem 1.1.

Since the boundary $\partial M$ is a compact sub-manifold in $M$ of codimension 1 , we can take $\lambda$ sufficiently large such that there exists the boundary normal coordinate chart on the boundary layer $L_{3 / \lambda}=\{p \in M: d(p, \partial M) \leq 3 / \lambda\}$ with respect to the boundary $\partial M$ (cf Hörmander $[8$, p. 51]). In particular, we have the map

$$
\mathscr{B}: \partial M \times[0,3 / \lambda] \rightarrow \mathrm{L}_{3 / \lambda}, \quad\left(\mathrm{p}^{\prime}, \delta\right) \mapsto \mathscr{B}\left(\mathrm{p}^{\prime}, \delta\right)
$$


such that $\delta \mapsto \mathscr{B}\left(\mathrm{p}^{\prime}, \delta\right), \delta \in[0,3 / \lambda]$, is the geodesic with arc-length parameter normal to $\partial \mathrm{N}$ at $\mathrm{p}^{\prime}$. Moreover, for each point $\left(\mathrm{p}^{\prime}, \delta\right) \in \mathrm{L}_{3 / \lambda}$, we have $0 \leq \delta \leq 3 / \lambda$ and

$$
\mathrm{d}\left(\left(\mathrm{p}^{\prime}, \delta\right), \partial M\right)=\delta
$$

Denote by $\mathscr{R}(\mathrm{r})$ the following $\mathrm{n}$-dimensional rectangle in $\mathbf{R}^{\mathrm{n}}$ sitting at the origin and having size $r$,

$$
\mathscr{R}(r)=\left\{x=\left(x^{\prime}, x_{n}\right)=\left(\left(x_{1}, \ldots, x_{n-1}\right), x_{n}\right) \in \mathbf{R}^{n}:\left|\left(x^{\prime}, 0\right)\right|<r, 0 \leq x_{n} \leq r\right\} .
$$

For a point $\mathrm{q}$ on $\partial M$, denote by $\operatorname{Exp}_{\mathrm{q}}$ at $\mathrm{q}$ the exponential map on the sub-manifold $\partial M$. Since $\partial M$ is compact and $\lambda$ is sufficiently large, we may assume the existence of the geodesic normal chart for each metric ball of radius $3 / \lambda$ on $\partial M$.

We choose and fix a point $p_{0}$ in $L_{1 / \lambda}$, and write $p_{0}=\mathscr{B}\left(q_{0}, \delta_{0}\right)$, where $q_{0} \in \partial M$ and $\delta_{0} \in[0,1 / \lambda]$. We denote by $R\left(q_{0}, 3 / \lambda\right)$ the rectangle in $M$ sitting at $q_{0}$ and having size $3 / \lambda$,

$$
\left.R\left(q_{0}, 3 / \lambda\right)=\left\{\left(\operatorname{Exp}_{q_{0}}\left(x^{\prime}\right), x_{n}\right)\right):\left(x^{\prime}, x_{n}\right) \in \mathscr{R}(3 / \lambda)\right\}
$$

In this way, we identity the rectangle $R\left(q_{0}, 3 / \lambda\right)$ in $M$ sitting at $q_{0}$ and containing $p_{0}$ with the rectangle $\mathscr{R}(3 / \lambda)$ in $\mathbf{R}^{n}$. Thus we could look at $u$ and $v$ as functions in $\mathscr{R}(3 / \lambda)$.

We recall that $u_{\lambda}$ and $v_{\lambda}$ are the corresponding rescaled functions of $u$ and $v$, respectively, i.e.,

$$
u_{\lambda}(y)=u(y / \lambda), \quad v_{\lambda}(y)=v(y / \lambda) \quad \text { for all } y \text { in } \mathscr{R}(3)
$$

To prove (10), we need only show the following estimate,

$$
\left|(\nabla \mathfrak{u})\left(\mathrm{p}_{0}\right)\right|=\left|(\nabla u)\left(0, \delta_{0}\right)\right| \leq \mathrm{C}\left(\lambda\|\mathrm{u}\|_{\mathrm{L}^{\infty}(\mathscr{R}(3 / \lambda))}+\lambda^{-1}\|v\|_{\mathrm{L}^{\infty}(\mathscr{R}(3 / \lambda))}\right),
$$

which can be reduced to the equivalent rescaled version,

$$
\left|\left(\nabla u_{\lambda}\right)\left(0, \lambda \delta_{0}\right)\right| \leq C\left(\left\|u_{\lambda}\right\|_{L^{\infty}(\mathscr{R}(3))}+\lambda^{-2}\left\|v_{\lambda}\right\|_{L^{\infty}(\mathscr{R}(3))}\right), \quad 0 \leq \lambda \delta_{0} \leq 1 .
$$

where $u_{\lambda}$ and $v_{\lambda}$ are the the rescaling function of $u$ an $v$, respectively. Observe that $u_{\lambda}$ is the solution of the Poisson equation

$$
\sum_{i, j} \partial_{y_{i}}\left(g_{\lambda}^{i j} \sqrt{g_{\lambda}} \partial_{y_{j}} u_{\lambda}\right)=-\lambda^{-2} \sqrt{g_{\lambda}} v_{\lambda} \quad \text { in the interior of rectangle } \mathscr{R}(3)
$$

and satisfies the Neumann boundary condition, i.e.,

$$
\frac{\partial u_{\lambda}}{\partial y_{n}}=0 \text { on the portion }\left\{x \in \mathscr{R}(3): y_{n}=0\right\} \text { of the boundary } \partial \mathscr{R}(3) \text {. }
$$

We shall give two different proofs for (12). 
1st proof The idea is to reduce, by Fact 1 in the introduction and the even extension, the question to the interior gradient estimate (7), which has been proved by the maximum principle in the former subsection. By the geometric property of geodesic normal coordinate chart with respect to the boundary $\partial M$, we have

$$
g^{n n}\left(x^{\prime}, x_{n}\right)=1 \text { and } g^{j n}\left(x^{\prime}, x_{n}\right)=0 \text { for } j \neq n \text { in } R\left(q_{0}, 3 / \lambda\right) \text {, }
$$

which implies that

$$
g_{\lambda}^{n n}\left(y^{\prime}, y_{n}\right)=1 \text { and } g_{\lambda}^{j n}\left(y^{\prime}, y_{n}\right)=0 \text { for } j \neq n \text { in } \mathscr{R}(3) \text {. }
$$

\section{Setting}

$$
a_{i j}:=g_{\lambda}^{i j} \sqrt{g_{\lambda}} \quad \text { for } \quad 1 \leq i, j \leq n-1, \quad a_{n n}:=\sqrt{g_{\lambda}}
$$

and

$$
b_{i}:=\sum_{j=1}^{n-1} \frac{\partial a_{i j}}{\partial y_{j}} \quad \text { for } \quad 1 \leq i \leq n-1, \quad b_{n}=\frac{\sqrt{g_{\lambda}}}{\partial y_{n}},
$$

we can express the Poisson equation (13) as

$$
\sum_{1 \leq i, j \leq n-1} a_{i j} \frac{\partial^{2} \phi}{\partial y_{i} \partial y_{j}}+a_{n n} \frac{\partial^{2} \phi}{\partial y_{n}^{2}}+\sum_{1 \leq k \leq n} b_{k} \frac{\partial \phi}{\partial y_{k}}=h \quad \text { in } \quad \operatorname{Int}(\mathscr{R}(3)),
$$

where $\phi=u_{\lambda}$ and $h=-\lambda^{-2} \sqrt{g_{\lambda}} v_{\lambda}$.

Set

$$
\mathscr{S}(\mathrm{r}):=\left\{y=\left(y^{\prime}, y_{n}\right)=\left(\left(y_{1}, \ldots, y_{n-1}\right), y_{n}\right) \in \mathbf{R}^{n}:\left|\left(y^{\prime}, 0\right)\right|<r,\left|y_{n}\right| \leq r\right\}
$$

which is the union of rectangle $\mathscr{R}(r)$ and its reflection with respect to the hyperplane $\left\{y_{n}=0\right\}$. We denote by $\tilde{\phi}$ the even extension onto $\mathscr{S}(3)$ of the function $\phi$ defined on $\mathscr{R}(3)$, i.e.,

$$
\tilde{\phi}\left(y^{\prime}, y_{n}\right)=\left\{\begin{array}{lll}
\phi\left(y^{\prime}, y_{n}\right) & \text { if } \quad y_{n} \geq 0 \\
\phi\left(y^{\prime},-y_{n}\right) & \text { if } \quad y_{n}<0
\end{array}\right.
$$

We do the even extension to $h$ and the coefficients $a_{i j}, a_{n n}, b_{i}$ for $1 \leq i, j \leq n-1$, and denote the corresponding extension functions on $\mathscr{S}(3)$ by

$$
\tilde{h}, \widetilde{a_{i j}}, \widetilde{a_{n n}}, \widetilde{b_{i}} \text {. }
$$

However we do the odd extension to $b_{n}$,

$$
\widetilde{b_{n}}\left(y^{\prime}, y_{n}\right)=\left\{\begin{array}{lll}
b_{n}\left(y^{\prime}, y_{n}\right) & \text { if } & y_{n} \geq 0 \\
-b_{n}\left(y^{\prime},-y_{n}\right) & \text { if } & y_{n}<0
\end{array}\right.
$$

We shall see soon that the possible discontinuity of $\widetilde{b_{n}}$ on the portion $\mathscr{S}(3) \cap\left\{y_{n}=0\right\}$ would not cause any trouble. 
Thus, we obtain the following Poisson equation about $\tilde{\phi}$ with continuous coefficients

$$
\sum_{1 \leq i, j \leq n-1} \widetilde{a_{i j}} \frac{\partial^{2} \tilde{\phi}}{\partial y_{i} \partial y_{j}}+\widetilde{a_{n n}} \frac{\partial^{2} \tilde{\phi}}{\partial y_{n}^{2}}+\sum_{1 \leq k \leq n} \widetilde{b_{k}} \frac{\partial \tilde{\phi}}{\partial y_{k}}=\tilde{h} \quad \text { in } \quad \operatorname{Int}(\mathscr{S}(3))
$$

except that $\widetilde{b_{n}}$ is bounded and possibly discontinuous on the portion $\mathscr{S}(3) \cap\left\{y_{n}=0\right\}$. By Fact 1 in the introduction, which can be proved by simple calculus computation, we know that $\tilde{\phi}$ is $C^{2}$ in $\mathscr{S}(3)$. The only point which we should take care of is whether $\widetilde{b_{n}} \frac{\partial \tilde{\phi}}{\partial y_{n}}$ is an even continuous function in $\mathscr{S}(3)$ with respect to $y_{n}$. However, by the extension $\tilde{\phi}$ of $\phi$ and the Neumann boundary condition, i.e., $\frac{\partial \phi}{\partial y_{n}}=0$ on $\left\{y_{n}=0\right\} \cap \mathscr{S}(3), \frac{\partial \tilde{\phi}}{\partial y_{n}}$ is an odd $C^{1}$ function vanishing on the portion $\mathscr{S}(3) \cap\left\{\mathrm{y}_{\mathrm{n}}=0\right\}$. Since $\widetilde{\mathrm{b}_{\mathrm{n}}}$ is a bounded function in $\mathscr{S}(3)$ and is odd with respect to $y_{n}, \widetilde{b_{n}} \frac{\partial \tilde{\phi}}{\partial y_{n}}$ is a continuous function being even with respect to $y_{n}$ in $\mathscr{S}(3)$. Moreover, $\widetilde{b_{n}} \frac{\partial \tilde{\phi}}{\partial y_{n}}$ vanishes on the portion $\left\{y_{n}=0\right\} \cap \mathscr{S}(3)$. Therefore, we have reduced the proof of $(12)$ to the estimate for $\tilde{\phi}$ at the interior point $\left(0, \delta_{0} \lambda\right)$ of $\mathscr{S}$ (3) similar to (9) in the former subsection. The fact that the bounded coefficient $b_{n}$ is possibly discontinuous on the portion $\left\{y_{n}=0\right\} \cap \mathscr{S}(3)$ does not bring us any trouble of applying the weak maximum principle. See Gilbarg-Trudinger [6, (3.3), p.31] and the related comments.

2nd proof The idea is to use the same even extension as above and the interior $C^{1, \alpha}$ estimate Gilbarg-Trudinger [6, Theorem 8.32, p. 210]. Denote by $\tilde{g}$ the even extension of the Riemannian metric $\mathrm{g}$ on $\mathscr{R}(3 / \lambda)$ onto $\mathscr{S}(3 / \lambda)$. Then $\tilde{g}$ is a Lipschitz metric on $\mathscr{S}(3 / \lambda)$ with $C^{0,1}$ norm bounded by the $C^{1}$ norm of $g$. Denote the even extension of $u$ and $v$ on $\mathscr{S}(3 / \lambda)$ by $\tilde{u}$ and $\tilde{v}$, respectively. We claim that $\tilde{u}$ is a weak solution of the following Poisson equation

$$
-\frac{1}{\sqrt{\tilde{g}}} \sum_{i, j} \partial_{x_{i}}\left(\tilde{g}^{i j} \sqrt{\tilde{g}} \partial_{x_{j}} \tilde{u}\right)=\tilde{v} \quad \text { in } \operatorname{Int}(\mathscr{S}(3 / \lambda)) .
$$

That is, for each smooth function $\psi$ compactly supported in $\operatorname{Int}(\mathscr{S}(3 / \lambda))$, the following integral equality holds

$$
\int_{\operatorname{Int}(\mathscr{S}(3 / \lambda))} \sum_{i, j} \tilde{g}^{i j} \partial_{x_{i}} \tilde{u} \partial_{x_{j}} \psi d x=\int_{\operatorname{Int}(\mathscr{S}(3 / \lambda))}(-\tilde{v}) \psi d x .
$$

Actually, since $\partial \tilde{u} / \partial x_{n}=0$ on $\mathscr{S}(3 / \lambda) \cap\left\{x_{n}=0\right\}$, we find by the Green formula on Rie- 
mannian manifolds and the even extension of $u$ and $v$,

$$
\begin{aligned}
\int_{\operatorname{Int}(\mathscr{S}(3 / \lambda)) \cap\left\{x_{n}>0\right\}} \sum_{i, j} \tilde{g}^{i j} \partial_{x_{i}} \tilde{u} \partial_{x_{j}} \psi d x & =\int_{\operatorname{Int}(\mathscr{S}(3 / \lambda)) \cap\left\{x_{n}>0\right\}} \Delta \tilde{u} \psi d x \\
& =\int_{\operatorname{Int}(\mathscr{S}(3 / \lambda)) \cap\left\{x_{n}>0\right\}} \Delta u \psi d x \\
& =\int_{\operatorname{Int}(\mathscr{S}(3 / \lambda)) \cap\left\{x_{n}>0\right\}}(-v) \psi d x \\
& =\int_{\operatorname{Int}(\mathscr{S}(3 / \lambda)) \cap\left\{x_{n}>0\right\}}(-\tilde{v}) \psi d x
\end{aligned}
$$

Using the change of variable $x_{n} \mapsto-x_{n}$ and the above equality, we obtain

$$
\int_{\operatorname{Int}(\mathscr{S}(3 / \lambda)) \cap\left\{x_{n}<0\right\}} \sum_{i, j} \tilde{g}^{i j} \partial_{x_{i}} \tilde{u} \partial_{x_{j}} \psi d x=\int_{\operatorname{Int}(\mathscr{S}(3 / \lambda)) \cap\left\{x_{n}<0\right\}}(-\tilde{v}) \psi d x,
$$

where we also use $g^{i n}=0$ for all $i \neq n$. Summing these two equality yields (15). Recall that coefficients $\tilde{g}^{i j}$ is Lipschitz and $\tilde{v}$ is continuous on $\mathscr{S}(3 / \lambda)$. On the other hand, we have the rescaled version of equation (15), i.e., for each smooth function $\psi$ compactly supported in $\operatorname{Int}(\mathscr{S}(3))$

$$
\int_{\operatorname{Int}(\mathscr{S}(3))} \sum_{i, j} \tilde{g}_{\lambda}^{i j} \partial_{y_{i}} \tilde{u}_{\lambda} \partial_{y_{j}} \psi d x=\int_{\operatorname{Int}(\mathscr{S}(3))}\left(-\tilde{v}_{\lambda}\right) \psi d x .
$$

Thus, applying to it the interior $C^{1, \alpha}$ estimate in Gilbarg-Trudinger [6, Theorem 8.32, $\mathrm{p}$. 210], we obtain that for every $0<\alpha<1$,

$$
\left\|\tilde{u}_{\lambda}\right\|_{C^{1, \alpha}(\mathscr{S}(2))} \leq \mathrm{C}\left(\left\|\tilde{u}_{\lambda}\right\|_{C^{0}(\mathscr{S}(3))}+\lambda^{-2}\left\|\tilde{v}_{\lambda}\right\|_{C^{O}(\mathscr{S}(3))}\right)
$$

which implied the desire estimate (12).

Acknowledgements The last author would like to thank Professor Qing Han, Professor Xinan Ma, Professor Christopher D. Sogge and Professor Meijun Zhu for valuable conversation during the course of this work. Y.S. is supported in part by the National Natural Science Foundation of China (No. 10971104), B.X. by the National Natural Science Foundation of China (Grant No. 11271343) and Anhui Provincial Natural Science Foundation (Grant No. 1208085MA01). The last two authors are supported in part by the Fundamental Research Funds for the Central Universities (Grant No. WK0010000020 and No. WK0010000023).

\section{References}

[1] Brandt, A.: Interior estimates for second order elliptic differential (or finite difference) equations via the maximum principle, Israel J. Math., 7, 95-121 (1969) 
[2] Brüning, J.: Über knoten von eigenfunktionen des Laplace-Beltrami operators. Math. Z. 158, 15-21 (1975)

[3] Donnelly, H., Fefferman, C.: Nodal sets of eigenfunctions on Riemannian manifolds. Invent. Math. 93(1), 161-183 (1988)

[4] Donnelly, H., Fefferman, C.: Growth and geometry of eigenfunctions of the Laplacian. Analysis and partial differential equations, Lecture Notes in Pure and Appl. Math., vol. 122, pp. 635-655. Dekker, NY (1990)

[5] Duong, X. T., Ouhabaz, E. M. and Sikora, A.: Plancherel-type estimates and sharp spectral multipliers, J. Funct. Anal., 196, 443-485 (2002)

[6] Gilbarg, D., Trudinger, Neil S.: Elliptic partial differential equations of second order. Reprint of the 1998 edition. Classics in Mathematics. Springer-Verlag, Berlin (2001)

[7] Grieser, D.: Uniform bounds for eigenfunctions of the laplacian on manifolds with boundary. Comm. Partial Differential Equations, 27(7-8), 1283-1299 (2002)

[8] Hörmander, L. The Analysis of Linear Partial Differential Equations III, Corrected second printing, Springer-Verlag, Tokyo 1994;

[9] Ozawa, S.: Asymptotic property of eigenfunction of the Laplacian at the boundary. Osaka J. Math. 30, 303-314 (1993)

[10] Safarov, Yu., Vassiliev, D.: The Asymptotic Distribution of Eigenvalues of Partial Differential Operators. Translations of Mathematical Monograph, 155. American Mathematical Society, Providence, RI (1997)

[11] Seeger, A., Sogge, C. D.: On the boundedness of functions of pseudo-differential operators on a compact manifolds. Duke Math. J. 59, 709-736 (1989)

[12] Shi, Y.-Q., Xu, B.: Gradient estimate of an eigenfunction on a compact Riemannian manifold without boundary. Ann. Glob. Anal. Geom., 38(1), 21-26 (2010)

[13] Shi, Y.-Q., Xu, B.: Gradient estimate of a Dirichlet eigenfunction on a compact manifold with boundary. DOI:10.1515/FORM.2011.115, Forum Math., published online: 20/04/2011

[14] Smith, H. F.: Sharp $\mathrm{L}^{2} \rightarrow \mathrm{L}^{\mathrm{q}}$ bounds on the spectral projectors for low regularity metrics. Math. Res. Lett., 13(5-6), 967-974 (2006)

[15] Sogge, C. D.: Eigenfunction and Bochner-Riesz estimates on manifolds with boundary. Mathematical Research Letters, 9, 205-216 (2002)

[16] Xu, B.: Derivatives of the spectral function and Sobolev norms of eigenfunctions on a closed Riemannian manifold. Ann. Glob. Anal. Geom. 26(3), 231-252 (2004)

[17] $\mathrm{Xu}, \mathrm{X}$.: Gradient estimates for eigenfunctions of compact manifolds with boundary and the Hörmander multiplier theorem. Forum Mathematicum 21(3), 455-476 (2009) 
[18] Xu. X: Eigenfunction estimates for Neumann Laplacian and applications to multiplier problems, Proc. Amer. Math. Soc., Article electronically published on March 3, 2011.

[19] Zelditch, S.: Local and global analysis of eigenfunctions on Riemannian manifolds, In: Handbook of geometric analysis, no. 1, 545-658, Adv. Lect. Math. vol. 7. Int. Press, Somerville, MA (2008)

\section{Author information}

Jingchen $\mathrm{Hu}$, Wu Wen-Tsun Key Laboratory of Mathematics, USTC, Chinese Academy of Sciences. School of Mathematical Sciences, University of Science and Technology of China, Hefei 230026 China.

Yiqian Shi, Wu Wen-Tsun Key Laboratory of Mathematics, USTC, Chinese Academy of Sciences. School of Mathematical Sciences, University of Science and Technology of China, Hefei 230026 China.

Bin Xu, Wu Wen-Tsun Key Laboratory of Mathematics, USTC, Chinese Academy of Sciences. School of Mathematical Sciences, University of Science and Technology of China, Hefei 230026 China.

E-mail: bxu@ustc.edu.cn 